\title{
Mouse Models of Brain Cancer: Insight into the Cytopathogenesis of Glioma
}

\author{
S. Ghazi, ${ }^{*}$ M. Stark, ${ }^{*}$ P. Li, ${ }^{*}$ H.L. Moses, ${ }^{* *}$ and T.W. Abel*
}

Departments of *Pathology and ${ }^{* *}$ Cancer Biology, Vanderbilt University, *1161 21st Avenue South, MCN C2318, Nashville, TN 37232-2561

Glioblastoma multiforme is one of the most aggressive human cancers, and the most common primary, adult CNS neoplasm. Effective treatment strategies are lacking for these tumors, which carry a dismal prognosis. Transgenic mouse models have emerged as powerful tools for studying the biology of glioma [1]. Mice have been engineered to study the effects of manipulating genes and signaling pathways that are known to be mutated or aberrantly regulated in human glioma. We reported a novel transgenic mouse model in which conditional activation of the Ras pathway in the brain led to an intermediate-grade glioma [2]. The glioma showed histopathologic features that closely mimicked human tumors, and glioma cells expressed markers associated with neural stem/progenitor cells (NSPC). In addition, the mice showed marked expansion of the subventricular zone (SVZ), a NSPC population in the brain. This mouse model may be useful for studying the potential role of NSPC in gliomagenesis. More recently, through concomitant, conditional deletion of the p53 tumor suppressor gene, we have developed a novel model of glioblastoma in these mice. This presentation will focus on Cre-loxP technology for introducing genetic manipulations in the brain, and the types of experimental questions that may be addressed.

Conditional, transgenic mouse models allow targeting of glioma-relevant genetic manipulations to brain cells. One method involves mice that have been engineered to express the DNA recombinase, Cre, under the control of brain tissue-specific promoters (Fig 1). One such mouse strain, the $h G F A P-C r e$ mouse, expresses Cre protein in restricted brain cell types under the control of the human glial fibrillary acid protein $(h G F A P)$ promoter [3]. These mice are mated with a second genetically engineered mouse strain that carries a mutant form of the proto-oncogene K-ras $(K$ ras $^{G 12 D}$ ) [4]. This mutant form of $K$-ras is silenced by stop sequences that are flanked by loxP DNA sequences. Bigenic progeny of this cross ( $h G F A P-C r e / K-r a s{ }^{G l 2 D}$ mice) inherit both the GFAP-Cre and loxP-stop-loxP-K-ras ${ }^{G 12 D}$ alleles. Cre expression results in recombination at the loxP sites, excision of the stop sequence, and expression of $K-$ ras $^{G 12 D}$. The CNS cell types in which recombination occurs have been well characterized and include NSPC. Additional genetic alterations may be introduced through breeding strategies that incorporate additional conditional alleles.

We have now developed a mouse model of glioblastoma multiforme. p53 mutation is a common genetic alteration in human glioma specimens. Again using $h G F A P$-Cre mice, we deleted $p 53$ in concert with activation of $K-$ ras $^{G 12 D}$. The intermediate-grade gliomas induced by activation of the $K$ $\operatorname{ras}^{G 12 D}$ alone are converted, with concomitant deletion of p53, to high-grade, lethal gliomas (Fig 2). Microscopic examination of the brains of two-week-old, $h G F A P-C r e / K-r a s{ }^{G 12 D} / p 53^{\text {fllfl }}$ pups shows early tumor formation around the SVZ. Cultured astrocytes harvested from $h G F A P-C r e / K-$ $\operatorname{ras}^{G 12 D} / p 53^{f l / f l}$ pups and injected into the brain of adult mice formed lethal tumors at approximately 3 weeks post-injection (Fig 3). This model may be useful as a pre-clinical tool for studying novel glioma therapies. In addition, it will be possible to use this model to address basic questions about glioma biology, including the role of NSPC, and tumor-microenvironment interactions [5]. 
References

[1] J.T. Huse and E.C. Holland, Brain Pathology. 19 (2009) 132.

[2] T.W. Abel et al., Molecular Cancer Research. 7 (2009) 645.

[3] L. Zhuo et al., Genesis. 31 (2001) 85.

[4] E.L. Jackson, et al., Genes and Development. 15 (2001) 3243.

[5] This research was supported by a Vanderbilt Physician Scientist Development Award and NIH/NINDS (1K08 NS062107-01A1).

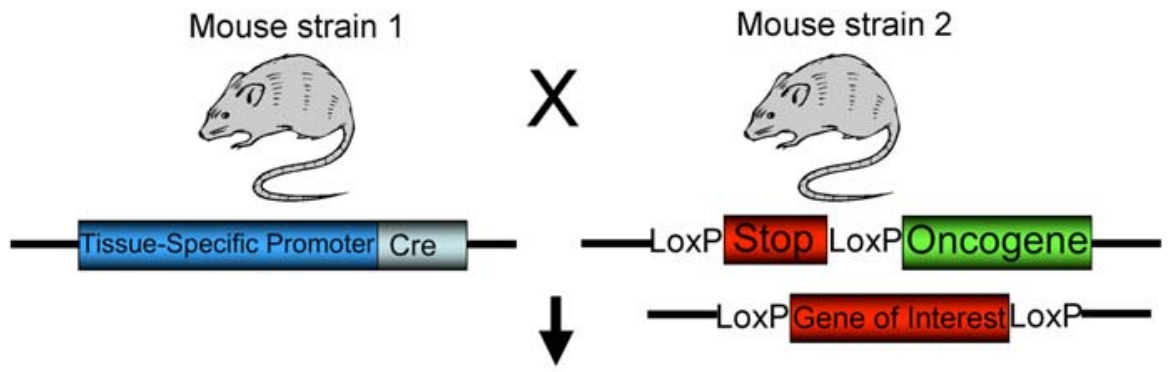

Mouse strain 3

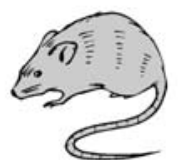

FIG. 1. Cre/LoxP systems may be used for tissue-specific activation of oncogenes and/or deletion of tumor suppressor genes.

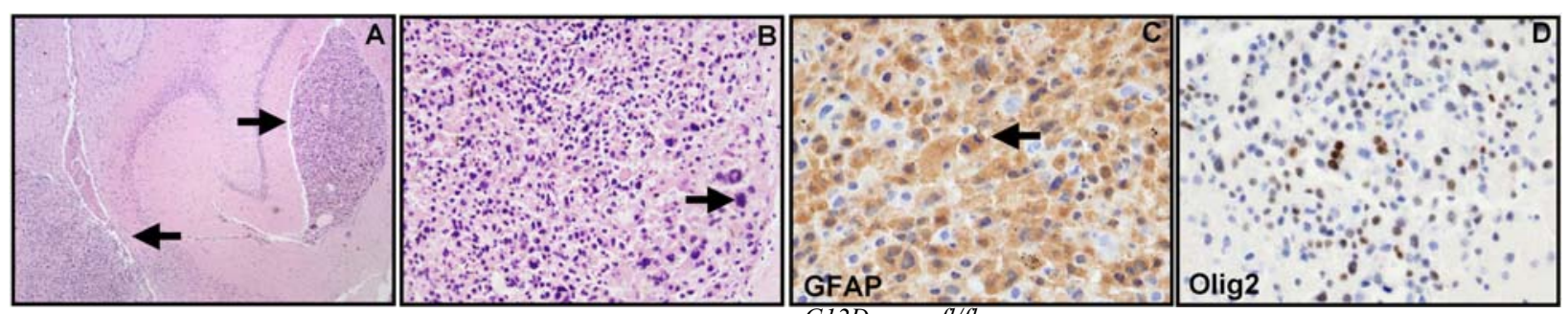

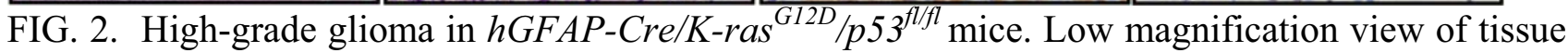
sections stained with H\&E (A). Arrows indicate tumor. Higher magnification of tumor focus (B). Note the large, pleomorphic cells (arrow). The cells express GFAP by immunohistochemistry (C). Note the mitotic figure (arrow). Tumor cells are immunoreactive for olig2 (D).
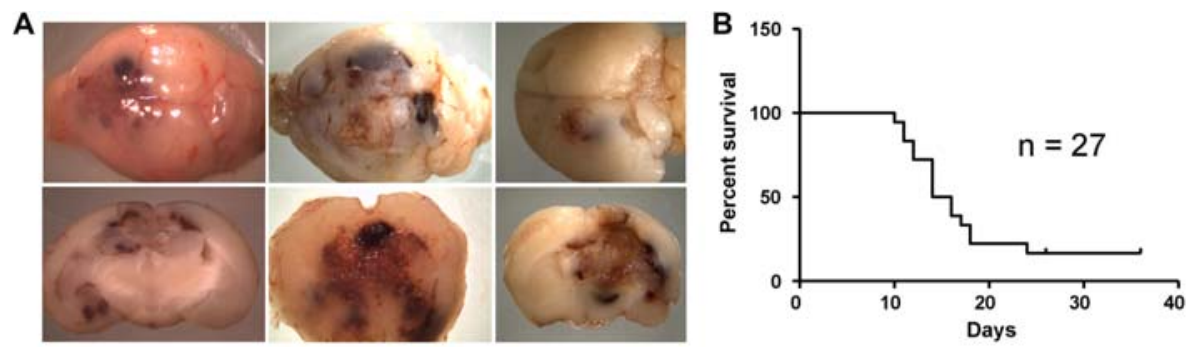

FIG. 3. Lethal, high-grade gliomas occur with short latency in mice transplanted orthotopically with

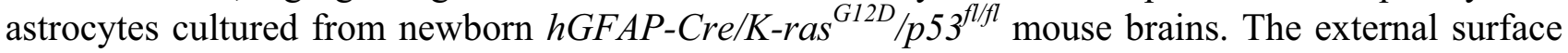
of the implanted brains showed hemorrhage (A, upper panels). Coronal sections showed a highly infiltrative tumor with hemorrhage and necrosis (A, lower panels). Survival curve shows that the majority of implanted mice succumb with tumor within 3 weeks of transplant (B). 